\title{
EFECTO DE LA ADMINISTRACION DE LA VITAMINA K SOBRE LA CURYA DE BILIRRUBINEMIA EN LA PRIMERA SEMANA DE VIDA DEL PREMATURO
}

\author{
Drs. JORGE E. HOWARD, CLARA ROMAN, INES EBENSPERGER, \\ PATRICIO OLIVOS, RACHEL R. PONTES.
}

\author{
Cátedta de Pediatria del Prof. De. A. Átiztía. Centro de Prematuros, \\ Hosp. L. Calvo Mackenna.
}

En el recién nacido de término y alimentado con leche humana se ha podido establecer que el nivel de protrombina inicialmente es bajo, en cifras de un $25 \%$ del normal para el adulto, valor que a continuación sigue una curva descendente que alcanza su máximo a los dos o tres días, con valor de solo un $10 \%$, para luego ascender y alcanzar su nivel primitivo a los seis o siete días.

Este descenso no es tan notorio si el recién nacido es alimentado con leche de vaca o derivados, ya que esta contiene una cantidad de vitamina $K$ cuatro veces superior, al tiempo que esta alimentación determina un rápido desarrollo de bacilo coli en el intestino, germen que como se sabe, contribuye a la síntesis de vitamina $K$. Por otra parte, este descenso en el valor de protrombina no se produce si a la embarazada se le aplica vitamina $K$ en los días previos al parto o bien si esta se le administra al recién nacido.

Por otra parte, la manifestación clínica más frecuente de la enfermedad hemorrágica del recién nacido, es la melena. Su aparición ocurre generalmente entre los 2 y 4 días de vida, coincidiendo con el momento en que el recién nacido tiene una exagerada hipoprotrombinemia. Este hecho permite pensar aue le enfermedad hemorrágica esté condicionada por esta hiponrotrombinemia. Es así como Dyggve ${ }^{1}$ señala que la incidencia de hemorragia es mayor en aquellos niños que muestran valores bajos de protrombina.

Concordante con estos hechos resultaba Iógico administrar vitamina $\mathbf{K}$ a todo recién nacido, con el obieto de subir la protrombinemia $y$ de este modo prevenir los accidentes hemorrágicos.

Sin embargo destués de un amplio uso de vitamina $K$, todavía se discute si esta terabia reduce o no la incidencia de enfermedađ hemorrágica del recién nacido. A pesar de no existir acuerdo sobre la eficacia de la administración de vitamina $\mathrm{K}$ al recién nacido de término, la opinión general es que esta debe darse a todo prematuro, por la mayor frecuencia de enfermedad hemorrágica en estos, frecuencia que fluctúa entre 1,6 a $4 \times 1000$ en los recién nacidos prematuros y sólo alcanza a $1 \times$ 1000 en Ios recién nacidos de término.

Las dosis recomendadas e inyectadas por vía intramuscular varían según diferentes investigaciones, $\mathrm{y}$ asi Harryment ${ }^{2}$ utiliza $5 \mathrm{mg}$ y Gairdner ${ }^{3}$ aconseja usar solo $0.5 \mathrm{mg}$ ya que para este último autor, con esta dosis se conseguiría igual efecto que con una dosis superior.

En época más o menos reciente se consideraba innocua la administración de vitamina $K$ al recién nacido $y$ así se llegó a dar dosis bastante más elevadas que las recomendadas: tal es el caso de clínicos como Hottinger ${ }^{4}$ que alcanzó a administrar una dosis total de $150 \mathrm{mg}$ de vitamina $\mathrm{K}$.

Ahora bien, últimamente han aparecido publicaciones europeas y norteamericanas en las cuales se llega a dudar seriamente sobre la innocuidad de la administración de estas sobredosis, con referencia especial al hecho de dar estas dosis elevadas al prematuro.

En efecto, diversos autores han hecho notar que la vitamina $K$ puede producir hiperbilirrubinemia, o bien, anemia hemolítica, la que secundariamente también produce un aumento de la bilirrubina circulante. Además, y solamente en fecha más reciente, se ha descrito el cuadro de Kernicterus del prematuro, entidad en que se produce la encefalopatía bilirrubinica, sin que exista incompatibilidad sanguínea y asociado a un aumento de la bilirrubina circulante. Estos dos hechos se han relacionado estrechamente, $y$ es asi que se ha asegurado que el empleo de do- 
sis elevadas de vitamina $K$ han producido un aumento en la incidencia del Kernicterus del prematuro.

En este trabajo se ha pretendido contribuir al conocimiento del efecto que la administración de altas dosis de vitamina $\mathrm{K}$ tiene sobre el nivel de bilirrubinemia en la primera semana de vida del prematuro.

\section{MATERIAL Y MÉTODO}

El material estuvo constituído por prematuros ingresados al Centro de Prematuros deI Hospital Luis Calvo Mackenna en el periodo comprendido entre el 11III-1957 y el 11-X-1957.

Los prematuros se seleccionaron al azar alternativamente, obteniéndose dos grupos: el Grupo en Estudio al cual se administró vitamina $\mathrm{K}$ y el Grupo Testigo al cual no se admínistró dicha vitamina.

En ambos grupos se clasificaron los niños de acuerdo a su peso de nacimiento en: niños de $1501 \mathrm{gr}$ de peso o más y ninos de menos de 1500 gr de peso.

A los prematuros del grupo en estudio, se les inyectó una dosis diaria de 10 $\mathrm{mg}$ de vitamina $\mathrm{K}$ * por vía intramuscular en sus tres primeros días de vida.

A los prematuros, tanto del grupo en estudio como del grupo testigo, se les hicieron determinaciones de bilirrubinernia diarias, durante los siete primeros días de vida. Para dicha determinación se empleó cl micrométodo de Hsia ", modificado para el colorimetro de Lange.

De este modo se analizaron 162 prematuros que se distribuyeron en la siguiente forma:

\begin{tabular}{|c|c|c|}
\hline Peso de nacimlento & Grupo en estudio & Grupo testigo \\
\hline $\begin{array}{l}\text { Con peso superior } \\
\text { a } 1501 \mathrm{gr} \text {. }\end{array}$ & 50 & 60 \\
\hline $\begin{array}{l}\text { Con peso tafertor } \\
\text { a } 1500 \mathrm{gr} \text {. }\end{array}$ & 22 & 30 \\
\hline Total de nlños & 72 & 90 \\
\hline
\end{tabular}

NOTA: Los grupos no resultaron iguales por haher tent do que eliminarse del estudio algunos nifios que fallecleron antes del 70 dia de vida a porque no se hicieron todas las bllirmibinemias requeridas en este estudilo.

- La vitamina K utilizada fué el producto comercia Eatin (Sanjtas) bisulfato de menadiona,
PROMEDIO LEAL DE LOS PESOS DE NACIMIENTO DE IOS PREMATUROS DEL GRUPO EN ESTUDIO $Y$ DEL GRUPO THSTIGO

\begin{tabular}{|c|c|c|}
\hline & Grupo en estudio & Grupo testigo \\
\hline & Peso promadlo & Peso promedio \\
\hline $\begin{array}{l}\text { Nifios con peso de } \\
\text { ngcimento superlor } \\
\text { a I501 } \mathrm{g}^{5}\end{array}$ & $1849 \mathrm{gr}^{r}$ & $1821 \mathrm{Er}$ \\
\hline $\begin{array}{l}\text { Ntfias con peso de } \\
\text { meclmiento inferlor } \\
\text { a } 1500 \text { gr }\end{array}$ & $1251 \mathrm{gr}$ & 1320 gr \\
\hline
\end{tabular}

Con el objeto de establecer si existían diferencias en los valores de bilirrubinemias encontrados, estos se analizaron estadisticamente para:

1. Conocer los promedios de bilirrubinemias en cada día de edad.

2. Estudiar en el total de las determinaciones, el porcentaje de bilirrubinemias iguales o superiores a $15 \mathrm{mg} \%$.

3. Apreciar el porcentaje de prematuros con bilirrubinemias iguales o superiores a $16 \mathrm{mg} \%$.

4. Analizar el porcentaje de bilirrubinemias iguales a superiores a $15 \mathrm{mg} \%$.

4. Analizar el porcentaje de bilirrubinemia iguales o superiores a $15 \mathrm{mg} \%$ en cada día de edad.

\section{RESULTADOS}

Al analizar los promedios de bilirrubinemia en el total de los prematuros, prescindiendo del peso de nacimiento, se desprende que los promedios de bilirrubinemia del grupo en estudio son siempre mayores que los del grupo testigo, pero

TABLA I

PROMEDIO LE LOS VALORES DE BUIRRUEINEMIA EN LOS 7 PRIMLEROS DIAS DE VIDA EHN LA TOTALIDAD DE LOS PREMATUROS

Influencla de la administración de la vitamina $\mathbf{B}$.

Centro de Prematuros - Hospital Luls Calvo Mackenna. 1957

\begin{tabular}{|c|c|c|c|c|c|}
\hline \multirow{3}{*}{$\frac{\begin{array}{c}\text { Edad en } \\
\text { dies }\end{array}}{1}$} & \multirow{2}{*}{\multicolumn{2}{|c|}{$\begin{array}{l}\text { Grupo en estudio } \\
\text { N. Det. Prom. } \\
\begin{array}{l}\text { Bill. } \\
\text { (mg }\end{array}\end{array}$}} & \multicolumn{2}{|c|}{ Grupo testigo } & \multirow{2}{*}{$\begin{array}{l}\text { Dif. de los } \\
\text { promedo }\end{array}$} \\
\hline & & & $\begin{array}{l}\text { N, Det } \\
(\%)\end{array}$ & $\begin{array}{c}\text { Prom. } \\
\text { Bll, } \\
\text { (m) }\end{array}$ & \\
\hline & 34 & 4,1 & 32 & 3,9 & 0,2 \\
\hline 2 & 54 & 5,5 & 61 & 5,5 & 0 \\
\hline 3 & 51 & 7.8 & 71 & 7,3 & 0.5 \\
\hline 4 & 56 & 10,1 & 78 & 9.4 & 0,7 \\
\hline 5 & 57 & 11,6 & 77 & 10,8 & 0,8 \\
\hline 6 & 33 & 13,2 & 67 & 12,1 & 1,1 \\
\hline 7 & 57 & 13.7 & 74 & 12,2 & 1,5 \\
\hline
\end{tabular}


las diferencias no son estadísticamente significativas. (Gráfico I).

\section{TABLA II}

PROMTRIO DE LOS VALORES TRE RILIRRUSINENIA EN LOS T PRIMEROS DIAS DE VIDA EN PREMATUROS CON FESO DF NACIMIENTO SUPERIOR A 1501 GT

Influencja de la admindstracfón de la vitamlon $\mathrm{K}$. Centro de Prematuros - Hospital Luio Calvo Mackentad.

\section{7}

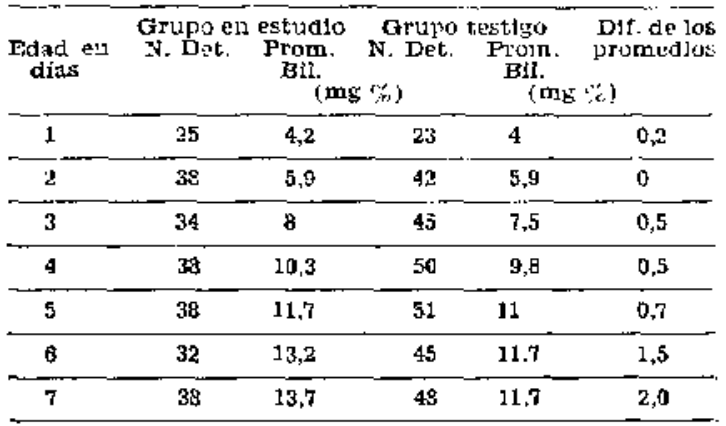

Se observa qua los promedios de las bilirrubinemias en los prematuros con peso de nacimiento superior a $1501 \mathrm{~g}$, son mayores en el grupo en estudio que en el grupo testigo. Sin embargo las diferencias no son estadísticamente significativas. (Gráfico II).

TABLA III

PROMEDIO DE IOS VALORES IYE BILIRRUBINEMTA FN LOS 7 PRIMEROS DIAS DE VIDA RN PREMATUROS EO LOS 'T PRIMEROS DIAS DE VIDA EN PREMATUROS

Influencia de la admlnistraclón de la vitamina $K$, Cculso de Prematuras - Bosptal Litis Calvo Mackenna.

\section{7}

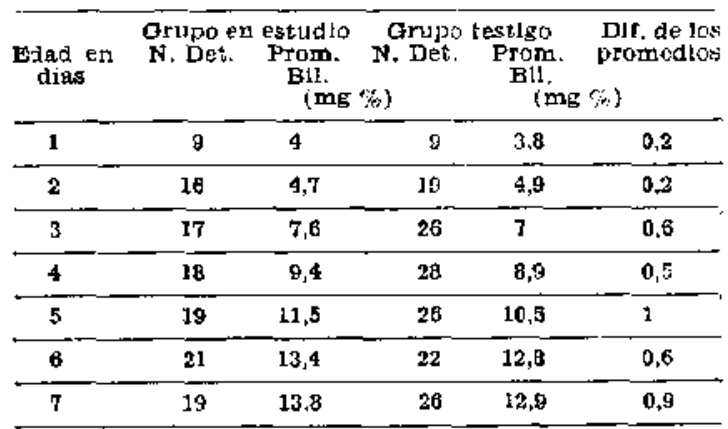

Los promedios de las bilirruminemias en los prematuros con pes? de nacimiento inferior a $1.500 \mathrm{~g}$, son mayores en el grupo en estudio que en el grupo testigo, pero las diferencias no tienen un valor estadistico significativo. (Gráfiço III).
TAELA IV

PORGENTAJE DE BILIRROBTNEMIA IGUALES O EUPERIORES A 15 mI DE VIDA, EN LA TOTALIDAD DE LOS PREMATUROS. Centro de Prematuros - Hospital Luls Calvo Mackenna.

I 957

\begin{tabular}{|c|c|c|c|c|c|}
\hline \multicolumn{6}{|c|}{ Bll. de $1.1 \mathrm{mg}$ o d superiorce } \\
\hline $\begin{array}{l}\text { Etad cul } \\
\text { dius }\end{array}$ & $\begin{array}{l}\text { Grupo en } \\
\text { No Det. }\end{array}$ & cotudio & $\begin{array}{l}\text { Grtipo } \\
\text { No Det. }\end{array}$ & istigo & $\begin{array}{c}\text { Wif. die los } \\
\text { poreentides }\end{array}$ \\
\hline 1 & 0 & 0 & 0 & 0 & a \\
\hline 2 & 0 & c & I & 1,6 & $--1,6$ \\
\hline 3 & 3 & 5.3 & 3 & 4,2 & 1.6 \\
\hline 4 & 5 & 3,9 & 8 & 10,2 & $-1,3$ \\
\hline 5 & $\begin{array}{l}10 \\
14\end{array}$ & $\begin{array}{l}17.5 \\
23.4\end{array}$ & $\begin{array}{l}11 \\
13\end{array}$ & $\begin{array}{l}14,3 \\
19,3\end{array}$ & $\begin{array}{l}3,2 \\
\$, 1\end{array}$ \\
\hline 7 & 22 & 39,5 & 15 & 20,2 & 18,3 \\
\hline $\begin{array}{l}\text { Total de } \\
\text { termina- } \\
\text { clopes }\end{array}$ & dE- 54 & 14,9 & 51 & $\$ 1,1$ & 3,8 \\
\hline
\end{tabular}

Analizando esta tabla se ve que los pcrcentajes de bilirrubinemias iguales a superiores a $15 \mathrm{mg} \%$ son mayores en el grupo en estudio. Las diferencias van aumentando del primero al séptimo dia de vida y en este último día hay una diferencia estadísticamente significativa. (Grático IV).

(7) dia: $\left.\frac{x}{T}=2,3\right)$.

T'ABLA V

PORCENTAJE DE BIIIRROELNEMUAS IGUALES O SUPERIORES A $15 \mathrm{~m} \%$ BN LOS 7 PRIMEROS DIAS DE VIDA, EN FREMATUROS CON PKSO DE NACJMIENTO SOPERIOR A $1501 \mathrm{gr}$.

Centro de Premeturos - Hospltal Lu1s Calvo Mackenini.

1957

\begin{tabular}{|c|c|c|c|c|c|}
\hline \multicolumn{6}{|c|}{ Bil. de $15 \mathrm{mg}$ 勧 o superiores } \\
\hline $\begin{array}{l}\text { Edad en } \\
\text { dlas }\end{array}$ & $\begin{array}{l}\text { Grupo en } \\
\text { No Det. }\end{array}$ & estudio & $\begin{array}{l}\text { Grupo } \\
\text { No Det. }\end{array}$ & $\underset{\%}{e x t i g o}$ & $\begin{array}{l}\text { Dir. de los } \\
\text { porcentr.jes }\end{array}$ \\
\hline 1 & 0 & 0 & 0 & 0 & 0 \\
\hline 2 & o & 0 & 1 & 2,4 & -2.4 \\
\hline 3 & 2 & 5,8 & 2 & 4,4 & 1,4 \\
\hline 4 & 5 & 13,1 & 6 & 12 & 1,1 \\
\hline 5 & 7 & 13,0 & 8 & 15,6 & 2,4 \\
\hline 3 & 9 & 23,1 & 6 & 13.3 & 14,8 \\
\hline 7 & 14 & 36,7 & $\mathrm{a}$ & 16,6 & 20,1 \\
\hline $\begin{array}{l}\text { Total de } \\
\text { tertnina } \\
\text { clones }\end{array}$ & 37 & 15.2 & 31 & 10.2 & 5,0 \\
\hline
\end{tabular}


Del análisis de esta tabla se desprende que los porcentajes de bilirrubinemias iguales o superiores a $15 \mathrm{mg} \%$ en los prematuros con peso nacimiento superior a $1501 \mathrm{gr}$, son mayores en el grupo en estudio, la diferencia en este porcentaje es mayor al $7^{\circ}$ día de vida y tiene valor estadfsticamente significativo. (Gráfico V). ( $7^{0}$ día de $\left.v_{1}-\frac{x}{T}=2,1\right)$.

TABLA VI

PORCFYTA.TE DE BLIRRUBINEMLAS IGUALES" $O$ SUPRRIORES $15 \mathrm{mF}$ EN LOS 7 PRIMEROS DIAS DE VIDA EN PEEMATUROS CON PESO DE NACIMIENTO INFERIOR A 1500 gT.

Centro de Prematuros - Hospltal Luis Calto Mackenne.

1957

\begin{tabular}{|c|c|c|c|c|c|}
\hline \multicolumn{6}{|c|}{ BH, de $15 \mathrm{~ms} \%$ o supertores } \\
\hline $\begin{array}{l}\text { Edad en } \\
\text { dlas }\end{array}$ & $\begin{array}{l}\text { Grupo en } \\
\text { No Det. }\end{array}$ & estudio & $\begin{array}{l}\text { Grupo } \\
\text { No Det. }\end{array}$ & estigo & $\begin{array}{l}\text { Dif, de los } \\
\text { porcentajes }\end{array}$ \\
\hline 1 & 0 & $\mathbf{0}$ & (6) & $\mathbf{0}$ & $\mathbf{0}$ \\
\hline 2 & 0 & 0 & $\mathbf{0}$ & a & $\mathbf{0}$ \\
\hline 3 & I & 5,8 & $\mathbf{1}$ & 3,8 & 2 \\
\hline 4 & 0 & 0 & 2 & 7,1 & $-7,1$ \\
\hline 5 & 3 & 15.7 & 3 & 15.2 & 0.5 \\
\hline 6 & 5 & 23,8 & 7 & 31.8 & $-8,0$ \\
\hline 7 & 8 & 42,1 & 7 & 26,9 & 15.2 \\
\hline
\end{tabular}

Total de de-

$\begin{array}{llllll}\begin{array}{l}\text { termina- } \\ \text { clones }\end{array} & 17 & 14,2 & 20 & 12,8 & 1,4\end{array}$

Se aprecia que existe un mayor porcentaje de prematuros en el grupo en estudio que presentan bilirrubinemias iguales o superiores a $15 \mathrm{mg} \%$, siendo las diferencias estadísticamente significativas. (Gráfico VI).

( ( Total de niños presenta- $\frac{x}{T}=2,1$ )

\section{Gráfico I}

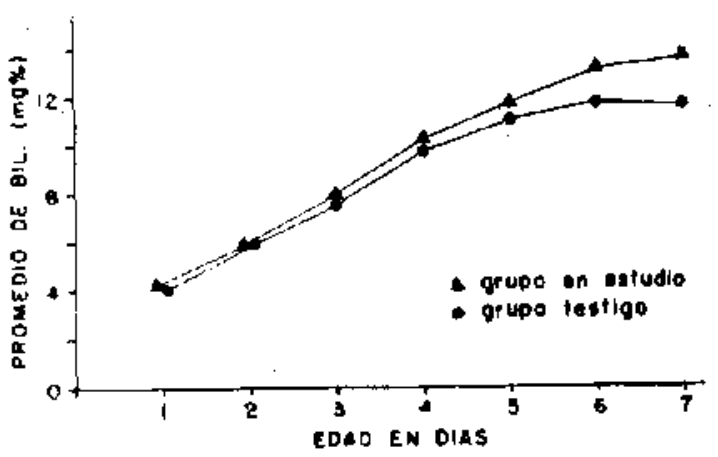

Gráfico II

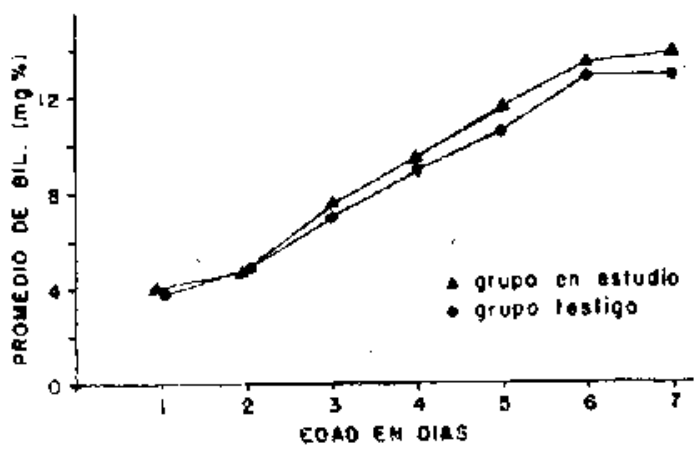

Gráfico III

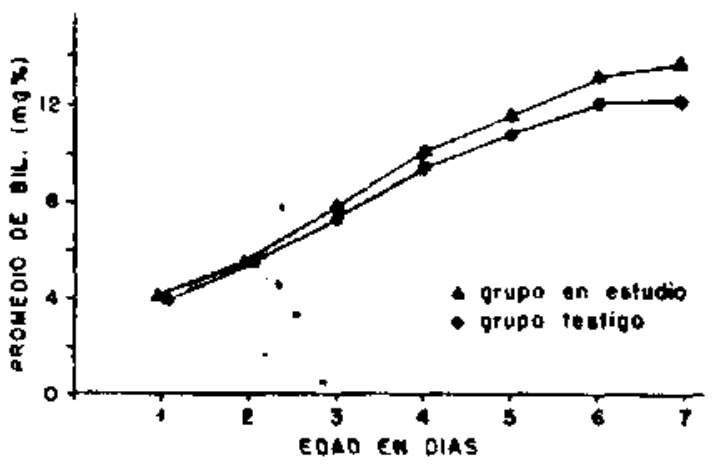

TABLA VII

PREMATUROS CON BILIRRUBLNGMAS IGUALES O SUPERIORES A 15 mB IEN ESTUDIO $\mathbf{Y}$ GRUPO THSTIGO.

Centro de Prematuros - Bospltal Luis Cajvo Mackenna. 1957.

\begin{tabular}{|c|c|c|c|c|c|c|c|}
\hline \multirow[t]{2}{*}{$\begin{array}{l}\text { Peso de } \\
\text { Nacimiento }\end{array}$} & \multirow[t]{2}{*}{ No de Diños } & \multicolumn{2}{|c|}{$\begin{array}{c}\text { en estudio } \\
\text { B11. } 15 \text { mo ro } \\
\text { superfores }\end{array}$} & \multirow[t]{2}{*}{ No de nurupos } & \multicolumn{2}{|c|}{ 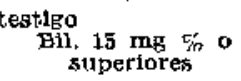 } & \multirow[t]{2}{*}{$\begin{array}{l}\text { Diferencias } \\
\text { de Jos } \\
\text { porcentejes }\end{array}$} \\
\hline & & Nơ & $\%$ & & $\mathbf{N} 9$ & $\%$ & \\
\hline Superlor a 1501 gr & 50 & 17 & 34,0 & 60 & 10 & 16,6 & 17,4 \\
\hline Inferior a $1500 \mathrm{gP}$ & 22 & 9 & 40,8 & 30 & 8 & 30 & 10,9 \\
\hline Total de nlffos & 72 & 26 & 36,1 & 90 & 18 & 21,1 & 15,0 \\
\hline
\end{tabular}


Se aprecia que los porcentajes de bílirrubinemias iguales o superiores a 15 $\mathrm{mg} \%$ en los prematuros con peso inferior del grupo en estudio, existiendo la mayor a $1500 \mathrm{gr}$ son mayores en los prematuros diferencia en el $7^{\circ}$ día, diferencia que no tiene valor estadístico significativo.

(7) dia: $\left.\frac{x}{T}=1,4\right)$.
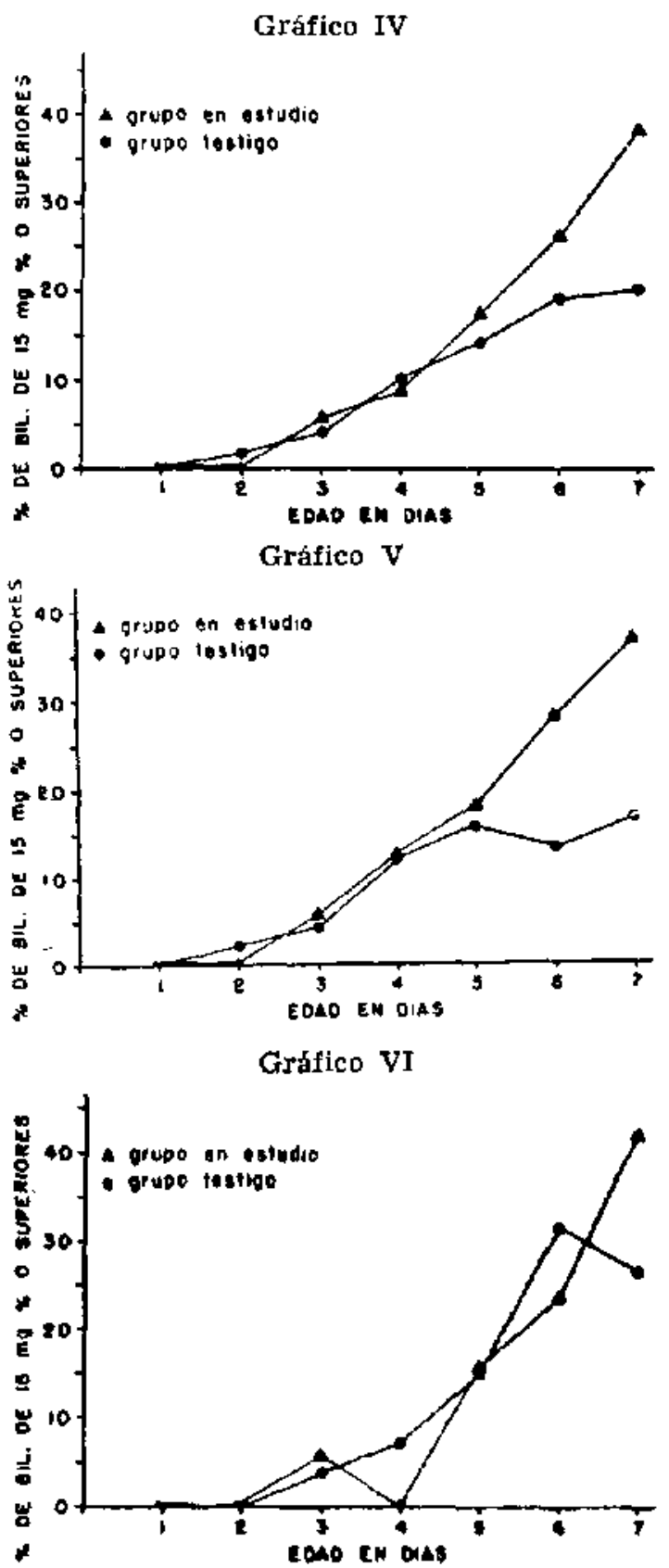

\section{COMENTARIO}

Experiencias realizadas en 1940 por Molitor y Robinson " señalaron que en ratas la administración de dosis masivas de vitamina $K$ determinaba la aparición de una notable disminución de los glóbulos rojos y hemoglobina. Igual conclusión obtienen Moore y Sharman en $1955^{7}$ en ratas sometidas a una dieta carenciada en vitamina $\mathrm{E}$, a las que administran dosis elevadas de vitamina $K$, determinando la aparición de una intensa hemolisis.

Gasser en 1953 * publica un estudio efectuado en 14 prematuros a los que administra vitamina $K$. Atribuyó a este agente un rol etiológico en ra aparición de una intensa anemia hemolítica. Igual fenómeno observó Allison en $1955^{\text {" estu- }}$ diando prematuros de pocos días de edad a los que dió dosis masivas de vitamina K. Ambos autores comprobaron un evidente aumento de la bilirrubina indirecta circulante.

Meyer y Angus en $1956^{\text {It }}$ publican Ios resultados de una investigación efectuada en recién nacidos $y$ en los que estudian las cifras de bitirrubinemia. El estudio incluyó 106 recién nacidos de término, de Ios cuales 46 recibieron al nacer $10 \mathrm{mg}$ de vitamina $K$ y los 60 retsantes constituveron el grupo testigo. Un estudio similar hicieron en 93 prematuros. De estos, 47 recibieron vitamina $K$ en dosis promedio de $30 \mathrm{mg}$. dejanto a 46 prematuros como testigos. Del análisis de sus resultados se desprende que aunque habia una gran dispersión de los valores de las bilirrubinemias encontradas en cada grupo, los valores medios de las bilirrubinemias entre los aue recibieron vitamina $\mathrm{K}$, ya fueran recién nacidos de término o prematuros, fueron superiores a los valores encontrados en los recién nacidos del grupo testigo, $v$ que no recibieron vitamina $K$.

Bound ${ }^{1 t}$ presenta resultados similares en el Congreso Internacional de Copenhague en 1956. mostrando que entre 55 prematuros a los que administró 30 $\mathrm{mg}$ de vitamina $\mathrm{K}$ (10 mg diarios intramuscular por tres días seguidos). 21 de ellos $\left(38 \%\right.$ ) tuvieron al $5^{0}$ día de vida niveles de bilirrubina circulante suberiores a $18 \mathrm{mg} \%$ y aue solamente $2(4 \%)$ de 51 prematuros a los que dió solo $1 \mathrm{mg}$ de vitamina $\mathrm{K}$ alcanzaron a sobrepasar dicha 
cifra de bilirrubinemia. El valor promedio de bilirrubinemia en el grupo que recibió $30 \mathrm{mg}$ de vitamina $\mathrm{K}$ fué de 15,4 mg\%, y esta cifra fué solo de $9,7 \mathrm{mg} \%$ en el grupo que recibió $1 \mathrm{mg}$ de vitamina $\mathbf{K}$, diferencia esta, considerada significativa.

Hottinger ${ }^{4}$ en el mismo año, compara las cifras de bilirrubinemia de un grupo de 106 prematuros a los que administró vitamina $K$ en dosis variables de 10 a 50 mg con otro grupo de 105 prematuros y a los que dió dosis fluctuantes de vitamina $\mathrm{K}$, entre 40 y $150 \mathrm{mg}$. Del análisis de sus resultados se desprende que no hubo diferencia significativa en los valores de bilirrubinemia de los dos grupos.

Es de pensar que estos resultados se debe al hecho de haber dado en ambos grupos dosis de vitamina $K$ que se pucden considerar masivas, careciendo por la tanto, de un verdadero grupo testigo que no hubiere recibido vitamina $\mathbf{K}$, o al menos, le hubiere sido administrada en dosis mínima.

Rossier ${ }^{12}$ en 1957 tampoco encuentra diferencias en las cifras de bilirrubinemias, pero en su publicación no indica las cantidades de vitamina $K$ empleadas.

Asteriadou-Samartzis y cols. en $1958^{13}$ estudian las modificaciones en los valores de bilirrubinemias en el $3^{\circ}$ día de vida de recién nacidos de término y en el $5^{\circ}$ día de recién nacidos prematuros, a los que suministran vitamina $K$ o vitamina $\mathrm{K} l$,agentes que les fueron administrados por distintas vías. Los resultados señalan que en aquellos prematuros que recibieron $30 \mathrm{mg}$ de vitamina $\mathrm{K}$ (10 $\mathrm{mg}$ diarios por tres dias) por vía intramuscular, las bilirrubinemias fueron significativamente más elevadas que las de los prematuros que no recibieron esta terapia o que solo se les administró $1 \mathrm{mg}$. de vitamina $K$. El valor promedio pudo ser mucho más elevado, si previo al $5^{\circ}$ día, no se hubiera efectuado en algunos de estos prematuros una exanguíneo transfusión, con el objeto de atenuar la concentración de bilirrubina que amenazaba subir exageradamente. En los prematuros y recién nacidos de término que recibieron $25 \mathrm{mg}$ de vitamina $\mathrm{Kl}$ endovenosa en una sola dosis, la concentración media de bilirrubina fué más baja que en el grupo testigo. Finalmente, cuando se utilizó la vía oral, administrándose $25 \mathrm{mg}$ de vitamina $\mathrm{Kl}$ en una sola dosis, la concentración media de bilirrubinemia fué aproximadamente la misma que en los testigos.

La vitamina $K$ podría actuar exclusivamente produciendo una hemolisis y secundariamente hiperbilirrubinemia de tipo indirecto, que el higado inmaduro del prematuro sería incapaz de escretar. Es probable que no sea éste el único mecanismo que intervenga en la producción de la hiperbiliurubinemia en el prematuro, ya que es posible, como lo aseguran Meyer y Angus, (10, que estas dosis masivas tengan una acción hépato-tóxica directa, $y$ en consecuencia, estos dos mecanismos: hemolisis y daño hepático, callsados ambos por la Vitamina $\mathrm{K}$, serán los responsables de esta hiperbilirrubinemia.

El análisis de los resultados expuestos en este trabajo, considerando Ia totalidad de los casos estudiados, nos permite concluir que los valores de bilirrubinemia son más elevadas en el grupo de estudio, pero que este aumento no es estadísticamente significativo. (Tabla I). Igual conclusión se obtiene al hacer el análisis de los casos según su peso de nacimiento (Tabla II y III).

De este modo, al analizar todo el material se puede suscribir ampliamente lo anotado por Meyer y Angus ${ }^{10}$ en el sentido que existe gran superposición de los valores individuales de bilirrubinemia en ambos grupos, pero que se encuentran valores medios de bilirrubinemia más elevados entre los que recibieron vitamina $K$.

Por otra parte, al considerar sólo aquellos prematuros que en algún momento del período de estudio hicieron bilirrubinemia iguales o superiores a $15 \mathrm{mg} \%$, y tomando como medida el porcentaje de determinaciones, es posible apreciar que hay un mayor porcentaje de bilirrubinemias altas entre los que recibieron vitamina $K$, sin que estas diferencias sean estadísticamente significativas. (Tabla IV).

Además en nuestro análisis es posible apreciar que del total de 72 prematuros que recibieron vitamina $K, 36,1 \%$ hicieron bilirrubinemia sobre $15 \mathrm{mg} \%$ y que esta incidencia fué sólo de $21,1 \%$ en el grupo testigo que no recibió vitamina $K$ (Tabla V).

Por último, si se analizan los casos que tuvieron bilirrubinemias superiores $a$ 
$15 \mathrm{mg} \%$ y se sigue la evolución de esta a través de los 7 días comprendidos en este estudio, es posible apreciar que los porcentajes de bilirrubinemias sobre $15 \mathrm{mg}$ son más elevados al $7^{\circ}$ día en los prematuros que recibieron vitamina $K$ y este aumento tiene valor estadístico significativo. (Tabla VI). Esta diferencia estadisticamente significativa en el porcentaje de bilirrubinemia, se observa también al 7 día en aquellos prematuros de peso superior a 1501 gr y que recibieron vitamina K. (Tabla VII).

En los de peso inferior a $1500 \mathrm{gr}$, tarnbién se encuentra un claro aumento de los valores de bilirrubinemia al séptimo dia, pero como el número de observación es muy pequeño, el resultado no es estadisticamente aceptable. (Tabla VIII).

En consecuencia, si comparamos nuestros resultados con los publicados por $\mathrm{Me}$ yer y Angus ${ }^{10}$ o Bound $y$ Telfer ${ }^{1+}$, se puede concluir que la vitamina $\mathrm{K}$ administrada en dosis elevadas, es capaz de producir en numerosas ocasiones una elevación de la bilirrubinemia, aumento que por las conecciones que tiene con el Sindrome de Kernicterus, resulta potencialmente peligroso.

En el Centro de Prematuros del Hospital Luis Calvo Mackenna no se ha logrado reunir un material suficiente para apreciar si en verdad disminuye la incidencia del Kernicterus al disminuir las dosis de, vitamina K. Esta complicación se produce en un porcentaje inferior al $4 \%$ del total de ingresos a un Centro de atención de prematuros, lo que explica que se necesite acumular un número muy grande de ingresos para lograr una conclusión en tal sentido.

En resumen, de este trabajo se desprende que la vitamina $K$ dada en dosis de 30 mg, eleva la bilirrubinemia a niveles que pueden resultar peligrasos favoreciendo la aparición del $\mathrm{K}$. I.

Por otra parte no hay razón alguna que aconseje utilizar dosis mayores de $1 \mathrm{mg}$ de vitamina $\mathrm{K}$ diario, ya que Hardwicke ${ }^{15}$ ha demostrado que dicha dosis es suficiente para prevenir la enfermedad hemorrágica del recién nacido, y que en las casos establecidos de esta enfermedad, basta administrar $2 \mathrm{mg}$ de vitamina $K$ para normalizar las cifras de la protrombina.

Concordante con esta observación es que en la actualidad en el Centro de Pre. maturos del Hospital Luis Calvo Mackenna solo se administra $1 \mathrm{mg}$ de vitamina $\mathrm{K}$ diario intramuscular por tres días. Igual norma ha implantado el "Comite de el Prematuro" de la Asociación de Pediatría Británica, recomendando no sobrepasar la dosis de $1 \mathrm{mg}$ intramuscular diario "l. Así también la Asociación Americana de Pediatría en su publicación. "Normas y Recomendaciones para la atención del recién nacido en hospitales" dice textualmente: "la vitamina $\mathrm{K}$ en dozis de $2,5 \mathrm{mg}$ debe ser suministrada, por lo menos una vez a todos los prematuros. La sobredosificación de vitamina $\mathrm{K}$ puede ser dañina". ${ }^{17}$.

\section{RESUMEN}

Los autores estudian el efecto de la vitamina $\mathrm{K}$ en los niveles de bilirrubina durante la primera semana de vida en 162 prematuros, los cuales estuvieron divididos en dos grupos: uno que recibió 10 mgrs de vitamina $\mathrm{K}$ durante 3 días seguidos, y otro grupo control que no recibió vitamina $\mathrm{K}$.

De acuerdo con sus resultados se demuestra que:

1. Los valores promedios de bilirrubinemias son más elevados en el grupo que recibió vitamina $\mathbf{K}$, pero las diferencias con el grupo control no son estadísticamente significativas.

2. El porcentaje de prematuros que presentaron bilirrubinemias iguales o superiores a $15 \mathrm{mgrs} \%$, es mayor en el grupo que recibió vitamina $\mathrm{K}$, y este valor es estadísticamente significativo.

3. Al séptimo día de vida del prematuro, los porcentajes de bilirrubinemia son más elevados en el grupo que recibió vitamina K. Este hallazgo es estadísticamente significativo.

4. Habiendose estableçido por diversos autores que el síndrome de kernicterus del prematuro está asociado a una hiperbilirrubinemia, todo factor qus aumenta a esta es potencialmente peligro:3o. En consecuencia, se recomienda no administrar dosis superiores a 2,5 mgrs de vitamina $\mathbf{K}$ en los prematuros. 


\section{SUMMARY}

THE EFFECT OF VITAMIN $K$ ON THE BLIRRUBIN CURVE DURING THE FIRST WEEK OF LIFE IN PREMATURE INFANTS.

The authors studies the effect of vitamin $\mathbf{K}$ on the bilirrubin curve during the first week of life in 162 prematures who were divided in 2 groups: those who recived 3 daily dosis of $10 \mathrm{mgrs}$. of vitamin $K$, and a control group that did not recive any vitamin K. From their study they conclude:

1. The averages values of bilirrubin are higher in the group that recived 10 mgrs. of vitamin $\mathrm{K}$ on 3 succesive days than in the control group, but these differences are not stadisticaliy significant.

2. The percentage of prematures that presented bilirrubin levels of $15 \mathrm{mgrs}$. \% or higher, is greater in the group that recived vitamin $K$, and this value has a statistical' significance.

3 . On the seventh day of life percentage of bilirrubin are higher in the group that recived vitamin $K$, and this findings is also of statistical significance.

4. As different authors have established that kernicterus of the premature is associated with hiperbilirrubinemia, any substance that might increase this is potentially dangerous. Based on this premise the authors recomend not to give doses over $2.5 \mathrm{mgrs}$. of vitamin $\mathrm{K}$ to premature infants.

\section{BIBLIOGRAFÍA}

1.-DYGave, H. - Prophy]actic trentment with vitamin K. 6th Int. Congress of Pedint. 1950.

2.-HARRYMIENT-H.; PATERSON-Mit,- Cready-Pediatries-Ed- 1957

3,-GAIRDNRR, D. - The care of the newhorn intant. Recent Advances In Paediatrlcs. J. \& A. Churchll Ltd.-Londan, 1954.

4,-HOTTINGER, H. - LIcters nucleilre chez les promaturés. Journees du prematuré. Ecole du Pucriculture, Paris. 1956.

5.-HSIA, D. Y. $\mathbf{Y}$; BSIA, H. H. and GELLIS, S. B. -A micromethdi for Serum Billrubin. Journal of Lab. nnd Cl. Medi. 40;610, 1952.

6-MOLTTOR, H. and ROBINSON, H. J. - Oral and Parentera] Toxicity of Vitamina K1, Phthloco! fisd 2 Methpl 1,4, Naphthoquinane Proc. Boe. Exp. Soc. Exp. BloI and Med. (N. Y.), 43:125, 1940 .

7.-MOORE, T. and SHARMAN, I. M. - Dinger of v1tamin $K$ analogues to newborn. Lancet, 1:819. 1955 .

8.-GASSER, C. - Dle hamolytische Frtingeburtenanitwle rait spontaner rouenkitiperblldung. Helv, paediat. Acta, 8:491, 1903.
8.-ALLISON, A. C. - Danger of vitamin $\mathrm{K}$ to newborn Lancet $1: 669$. 1055 .

10.-MEYER. T. C.: ANGUS, J. - The effect of targe dosis of "Synkavit" in the newborn. Areh. of Dls. in Ohllahood, $31: 212$, 1956 .

11.-BODND. J. P. - Kermleterus and Vitamin $K$. Internacionai Pediatrle Congress Copenhasue, 1956.

12.-ROSSIER, A. - L'Ictere nucléalre du prematuré. Etudes Neo-Natales, 6:51, 1957.

13-ASTERIADOD-SAMARTZIS, E. and LEIKIN, S.The relation of Vitamin $\mathrm{K}$ to Hyperbllirrubineinia. Pediatrles, 21:397, 2958.

14.-EOUND, J. F. and TELWER. T. F. - Erfect of VItamtr $\mathbf{k}$ on plasmabinrubit level 111 preconture mfants: Laneet: 1:720, 1956.

15.-HARDWICKE, $\$$. H. - Studies on the minirga: effective dose of a water-6oluble vitamin $K$ substitute in the prepention of hypoprothrombinemsa in the newborn infant. J. of Pedlatries, 24:259, ISci4. 16.-ROLE OF VITAMIN $K$. - Danger of large dojes. Lancet 1:902-1956.

17.-Normas y Recomendaclones para la ntenclón del reclén nacido en hospltales. Acadetnls Anterlcana de Peçíatra. p. 64.

\section{DISCUSIóN}

Dr. Gantes: Reconoce que los pediatras han usado la vitamina $k$ en dosis altas $y$ a veces mantinidas. El trabajo presentado confirma las experiencias extranjeras y nos sugiere ser un poxo más cauteloso en las dosis. Convendrí. que estos hechos llegaran a conocimiento de los tocólogos debido a que ellos usan en general dosis de 10 mgrs.

Dr. Garrés: Considera que las dosis de vitamina $\mathrm{K}$ corricatemente usadas son excesivas. En el Hospital Roberto del Río han reducido las dosis y actualmente usan $2.5 \mathrm{mgrs}$. en una sola inyección.

De. Roselló: Manifiesta que después del quinto dia el alumento de bilitrubinemia no tiene importancia en el recién nacido de término. pero sí en el prematuro, donde puede producir kernicterus.

Dr. Garcia: Expresa que en este rrabajo se demues. tra la acción que tiene la vitamina K sobre la bilirrubinemia. Respecto a la aoción de la vitamina, esca sería sobre un aspecto de la cougulación. ya que hay tambien problemas de fragilidad vasculat. Astualmente tienden a bajarse las dosis de $10 \mathrm{mgrs}$ a $2.5 \mathrm{mg} r \mathrm{rs}$. $y$ es posible que se llegue a suprimir.

Prof. Steeger: Se reficre a la acción que tendria la vitamina $K$ sobre ta bitirrubinemia, fenómeno que no está ওữ bien aclarado.

Dr. Howard: Manifiesta que es convenjente no confundir los términos "hemorragia del recièn nasido" con la "Enfermedad hemorrágica", siendo esta última por hipoprotrembinemia y con tratamienco específico a base de vitamina K. Con referencia a la acción de esta vitamina solre los niveles de bilirrubinemia, no excó bien claro. Es posible que exista o se produca uns alteración dentro del parénquuima hepitío. 